\title{
Dark photons in the decay of a Higgs-like boson
}

\author{
Gennady Kozlov* \\ Joint Institute for Nuclear Research \\ E-mail: kozlov@jinr.ru
}

The couplings of the Standard Model sector to scale invariant degrees of freedom can open the possibility to search for dark photons (DP). The decay of the Higgs-like scalar boson into a photon and DP is studied. The latter is mediated by the derivative of the scalar dilaton, emerging of which is provided by spontaneous breaking of scale symmetry. The limits are set on the DP mass, the mixing strength between the photon and DP.

38th International Conference on High Energy Physics 3-10 August 2016

Chicago, USA

${ }^{*}$ Speaker. 


\section{Introduction}

The interest to search for Dark Matter (DM), in particular dark photons (DP) as a conformal portal to DM, is increasing from year to year, and still is a mystery puzzle both in cosmology and in particle physics. The hidden sector containing DP can be explored at low energies (see, e.g., refs. in [1]), and in collider experiments at high energies. Increasing of the energy enables one to test the approximate conformal sector with its couplings to the Standard Model (SM) fields. In theories where an exact conformal symmetry is spontaneously broken, the low energy effective theory contains a massless scalar boson, the dilaton. The nature of the dilaton couplings is governed by the requirement that conformal symmetry be realized non-linearly. To collider physics, it is important that at the loop level the dilaton has potentially enhanced couplings to gluons and photons compared to those of the SM Higgs. In contrast to the latter, the dilaton couples to gluons even before running any SM particles in the loop through the trace anomaly.

We start with the conformal anomaly relevant to the coupling scalar-vector-vector containing a scale (dilatation) current $K_{\mu}$ and two vector currents which are related to each other, and this anomaly reflects the violation of scale invariance in SM by quantum effects. Our basis is the extended group $S U(2)_{L} \times U(1)_{Y} \times U^{\prime}(1)_{B}$, where index $B$ is associated with a hidden vector sector containing DP $\bar{\gamma}$. The standard photon $\gamma$ may oscillate into $\bar{\gamma}$ where the latter would be a short-lived boson decaying to invisible neutrino-antineutrino pair, $\bar{\gamma} \rightarrow \bar{v} v$ or to light lepton-antilepton pair, $\bar{\gamma} \rightarrow l \bar{l}$. If the approximate conformal invariance is broken at scale $\Lambda=4 \pi f$, the low energy spectrum of states may contain a light dilaton with its vacuum expectation value (vev) $f$, a light Higgs doublet or both. In the absence of the gauge sector that breaks electroweak (EW) symmetry, we assume the breaking of conformal invariance at the scale $\Lambda$ triggers EW symmetry breaking at the scale $\Lambda_{E W}=4 \pi v<\Lambda$, where $v=246 \mathrm{GeV}$ is the vev of the Higgs boson $H$. The dilaton operator triggers the breaking of $S U(2)_{L} \times U(1)_{Y}$ gauge invarince through the dilaton mass operator. The scales $f$ and $v$ are different except for $H(f=v)$. DP mixes with $\gamma$ via the kinetic term $\sim \varepsilon F_{\mu v} B^{\mu v}$ with $\varepsilon$ being the mixing strength; $F_{\mu \nu}$ and $B_{\mu \nu}$ are the strength tensors of electromagnetic field $A_{\mu}$ and the field $B_{\mu}$ of DP, respectively. The basic object is the Dalitz-like decay $S \rightarrow \gamma \bar{\gamma}$, where $S$ should either be $H$ or the scalar dilaton.

We study DP which is within the reach of the LHC energy $\sqrt{s} \sim O(10 \mathrm{TeV})$. Recently, DP and the resonant monophoton signatures in Higgs boson decays have been studied at the LHC [2]. The $\varepsilon$ is predicted in various models with the values in the range $10^{-12}-10^{-2}$. In the low energy experiments the values of $\varepsilon$ in the window $10^{-7}-10^{-3}$ have been probed (see the refs. in [3]). If no excess events are found, the obtained results can be used to impose bounds on $\varepsilon$ as a function of DP mass. The production of $S$ is due to gluon-gluon fusion followed by the (heavy) quarks in the loop with final states $\gamma$ and $\bar{\gamma}$. In SM, the violation of conformal symmetry is understood through the non-vanishing divergence of $K^{\mu}$

$$
\partial_{\mu} K^{\mu}=\theta_{\mu}^{\mu}=\frac{\beta(g)}{2 g} G_{\mu \nu}^{a} G^{\mu v a}+\sum_{q} m_{q}\left[1+\gamma_{m}\left(g^{2}\right)\right] \bar{q} q,
$$

where $\theta_{\mu}^{\mu}$ is the trace of the energy-momentum tensor, $\beta(g)$ is the standard beta-function with the coupling constant $g, G_{\mu \nu}^{a}$ is the strength tensor of gluon field. The quark state $q$ is accompanied by the mass $m_{q}$, and $\gamma_{m}\left(g^{2}\right)$ stands for the anomalous dimension of the mass operator $\bar{q} q$. 
Because of the scale invariance, the operator relevant to $\bar{\gamma}$ may carry the features of an unparticle stuff [4] with the scaling dimension $d_{I R}=1+\delta(\delta<1)$ in the infra-red (IR). In this case, the energy spectrum $\omega_{\gamma \bar{\gamma}}=d \Gamma(S \rightarrow \gamma \bar{\gamma}) / d E_{\gamma}$ of the photon (with energy $E_{\gamma}$ ) has a continuous distribution spreading from zero to half of the dilaton mass $m_{S} / 2$. As $\delta \rightarrow 0^{+}$(from above), $\omega_{\gamma \bar{\gamma}}$ becomes more peaked at $m_{S} / 2$. The $\bar{\gamma}$ with $\delta=0$ is $\gamma$. Note that the unitarity constraint lower bound on vector operator dimension $d_{I R} \geq 3$ [5] does not use here because the operator of $\bar{\gamma}$ is neither gauge invariant, no the primary one. The decay of $\bar{\gamma}$ into SM particles is controlled by the relation between the mass gap $m_{\bar{\gamma}}$ of $\bar{\gamma}$ and the production threshold $m_{1}+m_{2}$ with the masses $m_{i}(i=1,2)$ of the decay products. If $m_{\bar{\gamma}}>m_{1}+m_{2}$ there is enough phase space that $\bar{\gamma}$ can decay into SM particles, otherwise DP may be associated with DM as a stable object.

In this paper, DP is considered in the framework of the gauge dipole field model which exhibits IR singularities. In Abelian Higgs model the breaking of the gauge symmetry implies the dipole singularity of the type $\delta^{\prime}\left(p^{2}\right)$ in two-point Wightman functions (TPWF), e.g., for the scalar or the gauge fields, satisfying the equations of motion of 4th order (see the refs. in [6]). Other classes of models exhibiting a $\delta^{\prime}\left(p^{2}\right)$ singularity are the Conformal Field Theory (CFT) models [7].

\section{Couplings and constraints}

In CFT the ultraviolet (UV) coupling of an operator $O_{U V}$ of dimension $d_{U V}$ to a dilaton operator $\bar{\sigma}$ at the UV scale $M$ has the form

$$
\frac{1}{M^{d_{U V}-2}}|\bar{\sigma}|^{2} O_{U V}
$$

which flows in the IR to coupling of the Higgs boson $H$ to DP operator $O_{I R}$ of dimension $d_{I R}$

$$
\text { const } \frac{\Lambda^{d_{U V}-d_{I R}}}{M^{d_{U V}-2}}|H|^{2} O_{I R}, \Lambda \sim O(f),
$$

when the scale invariance is almost breaking. Once $H$ acquires $v$, theory becomes nonconformal below the scale $\tilde{\Lambda}[8]$

$$
\tilde{\Lambda}=\left(\frac{\Lambda^{d_{U V}-d_{I R}}}{M^{d_{U V}-2}} v^{2}\right)^{\frac{1}{4-d_{I R}}},
$$

where the hidden sector becomes the standard particle one. For a typical energy $\sqrt{s}$ of a collider experiment $\tilde{\Lambda}<\sqrt{s}<\Lambda$ the constraint on $\varepsilon$ is

$$
\varepsilon<\frac{s^{d}}{\left(v^{2} M^{d-2}\right)^{2}},
$$

where $d$ is the dimension of the SM operator and there are no the dependence on $d_{U V}, d_{I R}$ and $\Lambda$. The signals of new physics with DP are increasing with energy, and would be seen if the values of $M$ are not too large. If the deviation from the SM is detected at the level of order 3\%, the DP would be visible at the LHC as long as $v<M<1000 \mathrm{TeV}$. In the decay $S \rightarrow \gamma \bar{\gamma}$ the gauge invariant operator structure is $O_{S M} O_{I R} \sim \varepsilon \bar{q} \gamma_{\mu} q S B^{\mu} M^{-1}$ and the relevant energy scale is the mass of the heavy (top) quark $q$ in the loop. Since the new effects beyond the SM is expected on the scale $M>v \sim O(0.3 \mathrm{TeV})$, we find $\varepsilon<3 \cdot 10^{-2}(d=4)$. If $\varepsilon \rightarrow 0$, the only decay $H \rightarrow \gamma \gamma$ is appropriate within SM. 
The calculation of the electromagnetic neutrino formfactor $(\mathrm{EM} v \mathrm{~F})$ [9], applied to the process $\bar{\gamma} \rightarrow v \bar{v}$, allows to estimate the DP mass $m$

$$
m \simeq m_{\mu}\left[3 \sqrt{2} \pi \bar{\alpha}^{-1} \sum_{l: e, \mu}\left(\ln \frac{\Lambda_{v}^{2}}{m_{l}^{2}}-\frac{1}{6}\right)^{-1}\right]^{\frac{1}{2}} .
$$

Here, $m_{l}$ is the mass of the charged lepton $l$ in the loop, $\Lambda_{v}$ is the cut off scale in the logarithmically divergent integral of EMvF. Using $\Lambda_{v}$ at the scale of the $Z$-boson mass, we obtain $m=0.83 \mathrm{GeV}$ for an electron and muon loops, and as the result one finds $\varepsilon=7.6 \cdot 10^{-3}$.

\section{Model}

The Lagrangian density (LD) of the Higgs-dilaton Abelian gauge model is

$$
L=L_{\varepsilon}+L_{\sigma}-\frac{1}{2} m^{2} B_{\mu} B^{\mu}+L_{S I},
$$

where the field $B_{\mu}$ mixes with $A_{\mu}$ by

$$
L_{\varepsilon}=-\frac{1}{2} \varepsilon F_{\mu v} B^{\mu v}-\xi\left(\partial_{\mu} A^{\mu}\right)\left(\partial_{v} B^{v}\right)+\bar{q}\left(i \hat{\partial}-m_{q}-g \hat{A}\right) q-I^{\mu}\left(B_{\mu}-\partial_{\mu} \sigma\right),
$$

$\xi=\varepsilon \bar{\xi}, \bar{\xi}$ is a real number, $g$ is dimensioneless coupling constant, $F_{\mu \nu}=\partial_{\mu} A_{v}-\partial_{\nu} A_{\mu}, B_{\mu \nu}=$ $\partial_{\mu} B_{v}-\partial_{\nu} B_{\mu}$ and $I_{\mu}$ is an auxiliary vector field. The subcanonical scalar field $\sigma(x)$ with zero dimension in mass units is the primary dilaton field which provides a control over UV and IR divergences. LD (3.2) is invariant under the restricted gauge transformations of the second kind

$$
A_{\mu} \rightarrow A_{\mu}+\partial_{\mu} \alpha, B_{\mu} \rightarrow B_{\mu}+\partial_{\mu} \alpha, \sigma \rightarrow \sigma+\alpha, q \rightarrow q e^{i g \alpha}, I_{\mu} \rightarrow I_{\mu}
$$

where $\alpha(x)$ obeys the equation $\square \alpha(x)=0\left(\square \equiv \partial_{\mu} \partial^{\mu}\right)$. The parametrization of the $\sigma$ couplings to quarks that are relevant for collider physics is

$$
L_{\sigma}=-\sigma \sum_{q^{\prime}}\left(m_{q^{\prime}}+x_{\sigma} y_{q^{\prime}} v\right) q^{\prime} \bar{q}^{\prime}
$$

where $x_{\sigma}=m_{\sigma}^{2} / f^{2}<1$ parametrizes the size of deviations from exact scale invariance; $m_{\sigma}$ is the mass of the dilaton; $y_{q}$ are 9 additional contributions to the Yukawa couplings. If SM is embedded in the conformal sector, the following condition ia evident

$$
\sum_{\text {light }} b_{i}+\sum_{\text {heavy }} b_{i}=0
$$

where $i$ carries either QCD or EW features of the coefficients $b_{i}$ of corresponding $\beta$-functions. The sum in (3.4) is splitted over all colored particles into sums over light and heavy states in the mass scale separated by $m_{\sigma}$. The dilaton contribution to the decay $S \rightarrow \gamma \bar{\gamma}$ corresponds to including the states lighter than that of the dilaton in the $\beta$-function coefficients.

The scale invariant sector of the theory contains the fields $H$ and $\bar{\sigma}=f \sigma$

$$
L_{S I}(x)=\frac{1}{2}\left[\left(\partial_{\mu} H\right)^{2}+\left(\partial_{\mu} \bar{\sigma}\right)^{2}\right]-\frac{\lambda}{4}\left(H^{2}-\beta^{2} \bar{\sigma}^{2}\right)^{2}-\frac{\eta}{4}\left(\bar{\sigma}^{2}-f^{2}\right)^{2} .
$$


The conformal symmetry is breaking explicitly by the mass term $\sim \sqrt{\eta} f<f$. If $\eta=0$ we deal with the dilaton having the flat direction. Within the equations of motion coming from (3.1), one approaches the dipole equation for the Hermitian virtual dilaton field $\bar{\sigma}(x)$

$$
\square^{2} \bar{\sigma}(x)=0,
$$

where $\square \bar{\sigma}=\left(f / m^{2}\right)[\xi \square(\partial \cdot A)-(\partial \cdot I)]$.

The TPWF for $\bar{\sigma}(x)$ has the form $\omega(x)=\langle\Omega, \bar{\sigma}(x) \bar{\sigma}(0) \Omega\rangle$, where the vacuum vector $\Omega$ is defined as $\bar{\sigma}^{(-)}(x) \Omega=0,\langle\Omega, \Omega\rangle=1$, where $\bar{\sigma}(x)$ is decomposed into negative-frequency (annihilation) and positive-frequency (creation) parts: $\bar{\sigma}(x)=\bar{\sigma}^{(-)}(x)+\bar{\sigma}^{(+)}(x), \bar{\sigma}^{(+)}(x)=\left[\bar{\sigma}^{(-)}(x)\right]^{+}$. The function $\omega(x)$ should be Lorentz-invariant, and the equation $\square^{2} \omega(x)=0$ is evident. The general solution is given by the following expansion [10]

$$
\omega(x)=b_{1} \ln \frac{l^{2}}{-x_{\mu}^{2}+i \varepsilon x^{0}}+b_{2} \frac{1}{x_{\mu}^{2}-i \varepsilon x^{0}}+b_{3}
$$

which is the distribution on the space $S^{\prime}\left(\mathfrak{R}^{4}\right)$ of temperate generalized function on $\mathfrak{R}^{4}$. The coefficients $b_{1}$ and $b_{2}$ in (3.6) will be defined later, while $b_{3}$ is an arbitrary constant. The parameter $l$ in (3.6), having the dimension in units of length, breaks the scale invariance under dilatation transformation.

The commutator for dilaton field is

$$
[\bar{\sigma}(x), \bar{\sigma}(0)]=2 \pi i \operatorname{sign}\left(x^{0}\right)\left[b_{1} \theta\left(x^{2}\right)+b_{2} \delta\left(x^{2}\right)\right],
$$

where $b_{1}$ and $b_{2}$ can be fixed from the canonical commutation relations $\left[A_{\mu}(x), \pi_{A_{v}}(0)\right]_{\mid x^{0}=0}=$ $i g_{\mu \nu} \delta^{3}(\vec{x})$ and $\left[\bar{\sigma}(x), \pi_{\bar{\sigma}}(0)\right]_{\mid x^{0}=0}=i \delta^{3}(\vec{x})$, respectively. Here, $\pi_{\bar{\sigma}}(x)$ and $\pi_{A_{\mu}}(x)$ are the conjugate momenta of $\bar{\sigma}(x)$ and $A_{\mu}(x)$, respectively. In order to find $b_{1}$ and $b_{2}$, we choose $I_{\mu}$ in the form $I_{\mu}=\varepsilon m^{2}\left(\partial_{\mu} \sigma-A_{\mu}\right)$ which is invariant under gauge transformations (3.3). Hence, we have the new term in LD (3.1), namely $-(1 / 2) \varepsilon F_{\mu \nu} B^{\mu \nu}+\varepsilon m^{2}\left(A_{\mu}-\partial_{\mu} \sigma\right)\left(B_{\mu}-\partial_{\mu} \sigma\right)$ which is Stueckelberglike [11] LD, where the $\varepsilon$ - mixing effect between vector fields $A_{\mu}$ and $B_{\mu}$ is included, and one of the field, $B_{\mu}$, has the mass $m$. The following equations of motion

$$
\begin{gathered}
\square A_{\mu}-a \partial_{\mu}(\partial A)+m^{2}\left(A_{\mu}-\varepsilon^{-1} B_{\mu}\right)=m^{2} \partial_{\mu} \sigma, a=1-\bar{\xi}, \\
\left(\square+\tilde{m}^{2}\right)(\partial B)=\tilde{m}^{2} \square \sigma, \tilde{m}^{2}=\bar{\xi}^{-1} m^{2}
\end{gathered}
$$

are important to find the solutions of the model considered here. The solution $B_{\mu}=\partial_{\mu} \sigma$ obtained for an arbitrary vector $I_{\mu}$ obeys Eq. (3.8) that leads to (3.5). The solution of Eq. (3.7) is explicitly given in the form

$$
A_{\mu}=C_{\mu}+\frac{1}{m} \partial_{\mu} \varphi-\frac{\bar{\xi}}{m^{3}} \partial_{\mu} \square \varphi
$$

where $\varphi=\left(1+\varepsilon^{-1}\right) m \sigma$ with $\left(\square+m^{2}\right) C_{\mu}=0, \partial_{\mu} C^{\mu}=0$ and $\left[C_{\mu}(x), \varphi(y)\right]=0$. One can easily find the equation of motion for dilaton field: $\square \sigma \simeq \varepsilon\left(\bar{\xi} \square / m^{2}+1\right)(\partial \cdot A)$, and the conformal symmetry is restored (free massless dilaton field) if the $(\bar{\gamma}-\gamma)$ mixing does disappear $(\varepsilon=0)$.

The propagator of the dilaton field $\bar{\sigma}(x)$ is

$$
\tau(x)=\langle\Omega, T[\bar{\sigma}(x) \bar{\sigma}(0)] \Omega\rangle=-b_{1}\left[\ln \left|\kappa^{2} x^{2}\right|+i \pi \theta\left(x^{2}\right)\right]+b_{2}\left[\frac{1}{x^{2}}+i \pi \delta\left(x^{2}\right)\right],
$$


where

$$
b_{1}=\frac{1}{(2 \pi)^{2}} \frac{f^{2}}{\bar{\xi}(1+\varepsilon)}, \quad b_{2}=\frac{-1}{2 \pi^{2}\left(1-m^{2} / f^{2}\right)}, \quad \kappa \sim l^{-1} .
$$

The propagator of $B_{\mu}$ field (DP) in four-momentum space is $\tilde{\tau}_{\mu v}(p)=p_{\mu} p_{v} \tilde{\tau}(p)$, where $\tilde{\tau}(p) \sim\left[\tilde{\tau}_{1}(p)+\tilde{\tau}_{2}(p)\right]$, with

$$
\begin{gathered}
\tilde{\tau}_{1}(p)=\frac{1}{\bar{\xi}(1+\varepsilon)} \lim _{l^{2} \rightarrow 0}\left[\frac{1}{\left(p^{2}-\imath^{2}+i \varepsilon\right)^{2}}+i \pi^{2} \ln \left(l^{2} \imath^{2}\right) \delta_{4}(p)\right], \\
\tilde{\tau}_{2}(p)=\frac{-1}{2\left(f^{2}-m^{2}\right)\left(p^{2}+i \varepsilon\right)},
\end{gathered}
$$

where the "strong gauge condition" $\partial_{\mu} \sigma(x)=B_{\mu}(x)$ was used. In case of no mixing between the real photon and DP, $\tilde{\tau}_{\mu v}(p)$ comes to the standard photon propagator.

\section{Conclusions}

The gauge and scale invariant model for DP solvable in 4-dimensional space-time is studied, where the DP field is massive. The interaction between DP and quarks is mediated by the divergence of the dilaton. The latter is a portal to the propagator of DP $\tilde{\tau}_{\mu v}(p)$ obeying the equation of motion $\square(\partial \cdot B)=0$. We estimated the upper limit for the mixing strength $\varepsilon<3 \cdot 10^{-2}$ in $S \rightarrow \gamma \bar{\gamma}$, where the main contribution is due to top-quark in the loop. This can be interpreted as the limit of the branching ratio $B R(S \rightarrow \gamma \bar{\gamma})$ which is just the rate of the two-photon decay of the Higgs boson in the SM as $\varepsilon=0$. We find that the DP mass $m$ is restricted by $3.3 \mathrm{GeV}$ from above, and the results with $\mathrm{EM} v \mathrm{~F}$ calculations gives $m=0.83 \mathrm{GeV}$ and $\varepsilon=7.6 \cdot 10^{-3}$. The decay mode $S \rightarrow \gamma \bar{\gamma}$ can be used at the LHC to probe the DP sector since the emitted energy of the single photon is encoded with measuring of the missing of the recoil DP.

\section{References}

[1] J.L. Feng et al., Phys. Rev. Lett. 117 (2016) 071803.

[2] E. Gabrielli et al., Phys. Rev. D89 (2014) 015008.

[3] S.N. Gninenko, Phys. Rev. D87 (2013) 035030; D89 (2014) 075008.

[4] H. Georgi, Phys. Rev. Lett. 98 (2007) 221601; Phys. Lett. B650 (2007) 275.

[5] G. Mack, Commun. Math. Phys. 55 (1977) 1.

[6] G. Kozlov, I.Gorbunov, Int. J. Mod. Phys. A26 (2011) 3987.

[7] A. Salam and J. Strathdee, Phys. Rev. 184 (1969) 1760; P. Furlan et al., Riv. Nuovo Cim. 8 (1985) 3.

[8] P. Fox, A. Rajaraman and Yu. Shirman, Phys. Rev. D76 (2007) 075004.

[9] R.A. Buchl and B.P. Nigam, Phys. Rev. D7 (1973) 1554.

[10] R. Ferrari, Il Nuovo Cim. 19A (1974) 204.

[11] E.C.G. Stueckelberg, Helv. Phys. Acta 11 (1938) 225. 